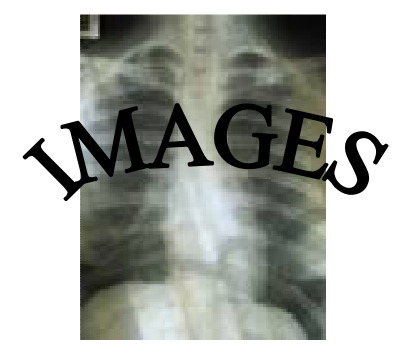

\title{
Unusual Cause of Gastric Compression:
} Pancreatic Pseudocyst

Georges C. Elhomsy, M.D. ${ }^{1}$

Edgard Wehbe, M.D. ${ }^{2}$

${ }^{1}$ The Lebanese University Faculty of Medical Sciences, Department of Internal Medicine. Beirut, Lebanon

${ }^{2}$ University of Kansas School of Medicine-Wichita

Department of Internal Medicine

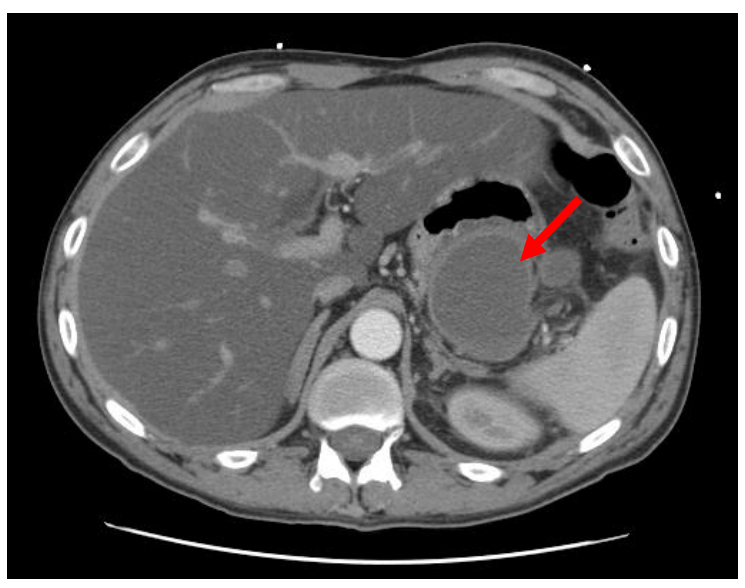

A 47-year-old male patient was admitted for a one-week history of nausea, vomiting, and abdominal discomfort. He had a partial gastrectomy secondary to peptic ulcer disease ten years earlier and was diagnosed with alcoholic pancreatitis one month prior to this hospitalization. The physical examination showed mild distention of the abdomen with tenderness on deep palpation of the epigastric region. Laboratory examinations revealed a hemoglobin of $11.6 \mathrm{~g} / \mathrm{dl}$, a potassium level of $2.6 \mathrm{meq} / \mathrm{L}$, and a lipase level of $331 \mathrm{U} / \mathrm{L}$. A contrast-enhanced CT of the abdomen showed multiple pseudocysts in the pancreatic bed and tail with significant mass effect on the stomach (see arrow). The patient was treated conservatively with resolution of symptoms after 72 hours. He was discharged on total parenteral nutrition and analgesics. Repeated CT of the abdomen six weeks later revealed complete resolution of the pancreatic pseudocyst.

Pancreatic pseudocyst is a well-recognized complication of pancreatitis occurring in $10 \%$ of the cases. ${ }^{1}$ Occasionally, the pseudocyst extends to distant areas within the abdominal cavity or may invade a nearby anatomic structure. Direct extension into the stomach infrequently is noted both radiographically and clinically. ${ }^{2}$ The thickening of the stomach wall offers resistance for direct compression or infiltration. In this patient, the partial gastrectomy and secondary weakening of the gastric muscle wall could be the major contributory factor for these unusual findings.

\section{References}

${ }^{1}$ O'Malley VP, Cannon JP, Postier RG. Pancreatic pseudocysts: cause, therapy, and results. Am J Surg 1985; 150:680-682.

2 Vitello JM. Gastric intramural pseudocyst with associated gastric outlet obstruction: recognition and management. South Med J 1996; 89:534-537.

Keywords: pancreatic pseudocyst, pancreatitis, gastrectomy, case report 